\title{
Gravitational Search for Designing a Fuzzy Rule-Based Classifiers for Handwritten Signature Verification
}

\author{
Marina Bardamova, Anton Konev, Ilya Hodashinsky, Member, IEEE and Alexander Shelupanov, \\ Member, IEEE
}

\begin{abstract}
Handwritten signatures are used in authentication systems as a universal biometric identifier. Signature authenticity verification requires building and training a classifier. This paper describes a new approach to the verification of handwritten signatures by dynamic characteristics with a fuzzy rule-based classifier. It is suggested to use the metaheuristic Gravitational Search Algorithm for the selection of the relevant features and tuning fuzzy rule parameters. The efficiency of the approach was tested with an original dataset; the type II errors in finding the signature authenticity did not exceed $0.5 \%$ for the worst model and $0.08 \%$ for the best model.
\end{abstract}

Index Terms-Authentication, Verification, Biometrics, Fuzzy Classifier, Gravitational Search Algorithm.

\section{INTRODUCTION}

$\mathrm{B}$ iometric authentication is used in security systems protecting information resources from unauthorized access, such as databases, software, operation systems, as well as in access control systems of physical assets, equipment, and territories. The biometrics allows eliminating the need to remember access passwords or to have physical identifiers like tokens, or cards. Besides, biometric data are more difficult to obtain and falsify.

There are two commonly accepted groups of unique personal biometric identifiers: physiological and behavioral [1]. The former includes static features, such as fingerprints, eye retina or iris, facial features, palm vein patterns, and the others. The latter group includes measurable dynamic features.

A person can be identified by voice, keyboarding or

Manuscript received December 20, 2018; revised July 26, 2019. Date of publication August 28, 2019. Date of current version August 28, 2019. The associate editor prof. Matko Šarić has been coordinating the review of this manuscript and approved it for publication.

This work was partially supported by the Ministry of Education and Science of Russia, Government Order no. 2.8172.2017/8.9 (TUSUR).

Authors are with the Department of Complex Information Security of Computer Systems, Tomsk State University of Control Systems and Radioelectronics, Russia.

E-mails: bmb@keva.tusur.ru, hia@keva.tusur.ru,kaa1@keva.tusur.ru, and saa@keva.tusur.ru.

Digital Object Identifier (DOI): 10.24138/jcomss.v15i3.678 handwriting, gait, and other characteristics.

Physiological identifiers are quicker and more convenient to use; however, they offer less authentication security. There are known situations when access systems were tampered with by using falsified physiological identifiers, or there were errors due to the high similarity of certain people, for example, relatives, or the access was denied for a sick or injured person [2-4]. Besides, authentication systems based on static parameters are rather expensive. Behavioral features expose the uniqueness of the individual, which is harder to falsify; therefore, dynamic features are considered to be more secure identifiers.

A handwritten signature is a historically proven and most frequently used authentication means. An authentication system based on handwritten signatures should include the following elements: a signature input device (usually a graphic tablet) and signature processing software that extracts features and recognizes the signature owner based on the identified features. It's necessary to use a classifier trained to define the authenticity of the signature and its owner for the verification procedure.

The most popular methods for data classification are decision trees, rule-based methods, probabilistic methods, support vector machines, and neural networks. Fuzzy rulebased classifiers are commonly nonlinear and this aspect is helpful in reducing possible classification error. Another important characteristic of fuzzy classifiers concerns their transparency. The transparency of classifiers constitutes a unique advantage of fuzzy classifiers and is linked with the interpretability of classification rules [5-7].

The process of fuzzy rule-based classifier design includes the following principal stages: feature selection, structure formation (rule base), and optimization of fuzzy rule parameters. Feature selection methods are conventionally grouped into two categories: filters and wrappers. The difference between filters and wrappers is whether the classifier is constructed during feature selection. The structure of the classifier is most often formed with the use of clustering methods designed to identify the data structure and build information granules that may be related to linguistic terms $[5$, 
8, 9]. Parameters of fuzzy rules can be optimized using conventional approaches based on calculation of derivatives or of metaheuristics methods [9-14].

This paper suggests a signature verification method based on the dynamic characteristics of handwritten signatures with a fuzzy rule-based classifier using the Gravitational Search Algorithm to select the relevant features and adjust the term parameters.

The rest of the article is organized as follows: Section II summarizes related works. Section III provides information about fuzzy-rule-based classifiers and performance of the fuzzy classifiers. Section IV briefly describes the algorithm for creating a fuzzy rules base. Section V explains process of feature selection and tuning fuzzy rule parameters with the gravitational search algorithm. Section VI presents the experimental results. Results and discussions are presented in Section VII. Conclusions are covered in Section VIII.

\section{RELATED WORK}

\section{A. Handwritten Signature Authentication}

There are two known methods of handwritten signature analysis: offline and online. The offline method is based on static features, such as the geometric proportion of the shape and the size. The shape is the consciously controlled aspect of a signature, and it is obviously not very hard to reproduce with due training. The online method implies analyzing the dynamic characteristics of the signature, such as pressure, inclination, coordinate change speed, etc. These features are non-conscious and more difficult to falsify or reproduce based on the signal pattern. Therefore, user authentication based on the dynamic characteristics of their signatures is considered the most effective [15].

The main approaches to analyzing the signature dynamics are as follows: the approach based on global features, the functional, regional, and hybrid approaches.

The first approach consists in forming global features from the entire signature image and local features from certain parts of the signature. The fuzzy system is used to verify dynamic signatures using global features in [16]. Feature selection is performed by a genetic algorithm, and for each signer an individual feature set of attributes is defined. Every feature is given weight, which is taken into account in the classification process.

Signature attributes (speed, acceleration, direction of movement of the pen, pressure) are represented as time series in a functional approach. Comparison is performed using measures of elastic distance, for example, dynamic transformation of the time scale [17-19].

The third approach uses the division of the signature signals into regions at the training and verification stage. The authors of [20] proposed a stroke-based algorithm that splits the speed into three bands. It was shown that only the medium-velocity band can be successfully used for recognition. The division of signatures into vertical and horizontal sections was described in [21]. The vertical sections corresponded to the initial, middle, and last moments of the signature execution time. The horizontal sections corresponded to the signature areas associated with high and low pen velocity and high and low pressure. Another approach is presented in [22], where the signature was divided into several sections. Each section had its own codebook and weight value. The final result was based on the merger of the evaluation results of each codebook.

The hybrid approach implies using a combination of the methods described above. The authors of [23] proposed an approach for verifying signatures based on the time series ( $\mathrm{x}$, y), similarity coefficients, and Hotelling statistics, which reduces the amount of data required for classification. Features and the similarity coefficients associated with them create new composite features, the use of which increases the accuracy of classification. An authentication system based on an ensemble of local, regional and global comparisons is presented in [24]. The following approaches were used: the merging of two local methods that use the Dynamic Time Warping and the approach based on the Hidden Markov Model, where each signature is described using its regional properties.

\section{B. Feature Selection}

The stage of feature selection is essential in order to build the least computationally sophisticated classifier. The least sophisticated the system, the faster the calculation. Besides, removing noise and excessive features allows enhancing the classification accuracy.

There are two main categories of the feature selection methods: wrappers and filters [25]. The wrapper method includes the classifier construction stage in the selection process and consists in searching for a feature subset based on the quality criterion of the built model. On the contrary, filters select features separately and are based on searching for and analyzing dependencies between the feature-based description and classes.

The wrapper methods demonstrate better results due to the close connection with the classifier. On the other hand, the need for multiple reconstructions of the classifier results in increasing training costs and can lead to the model retraining [26]. The strong side of filters is their universal application and low computational complexity of the filtering algorithms, while the weakness of this method consists in the fact that features are normally selected independently from each other [26].

In publication [26], it is noted that there is no single best method for feature selection and the efforts should be focused on seeking the suitable method for each specific problem.

\section{Gravitational Search Algorithm}

The metaheuristic Gravitational Search Algorithm was first proposed in 2009 [27]. In this algorithm, inspired by the algorithm of particle swarm optimization, the searcher agents are a collection of masses, which interact with each other based on the laws of Newton. The proposed method has been compared with some well-known heuristic search methods. The obtained results confirm the high performance of the proposed method in solving various nonlinear functions. It has been shown that the Gravitational Search Algorithm is able to find the optimum solution for many benchmarks. For this 
reason, this algorithm was chosen to solve the problem of designing a fuzzy rule-based classifier.

This article aims to describe the new authentication method based on the dynamic characteristics of handwritten signatures with a fuzzy rule-based classifier. The main contributions of this paper can be summarized as follows:

- The technique based on using a fuzzy rule-based classifier to identify the signature authenticity based on the dynamic characteristics of the signature obtained with a graphic tablet and converted into a feature vector by decomposing the signal sequences into a Fourier series.

- The classifier structure generation method based on fuzzy rules.

- The method of relevant feature selection for the fuzzy rule-based classifier by means of the binary Gravitational Search Algorithm with the V-shaped transform function acting as a wrapper.

- The criterion of the feature subset selection in case of class instance imbalance and a great number of features and classes, based on the statistical Akaike information criterion.

- The method tuning fuzzy rule parameters based on the Continuous Gravitational Search Algorithm.

- Experimental results show that our proposed method is effective.

\section{PROBLEM FORMULATION}

\section{A. Fuzzy Rule-based Classifier}

The classification consists in identifying the most suitable class for the object based on the features vector. The classification algorithm reproduces the dependence between the feature values and the set of classes based on the available retrospective records in the observation dataset.

In universal set $U=(A, C)$, where $A=\left\{x_{1}, x_{2}, \ldots, x_{n}\right\}$ is the set of input features, $C=\left\{c_{1}, c_{2}, \ldots, c_{m}\right\}$ is the set of classes, and $\mathbf{X}=x_{1} \times x_{2} \times \ldots \times x_{n} \in \mathfrak{R}^{n}$ is the $n$-dimensional space of features, the fuzzy rule-based classifier can be represented as a function that assigns a calculable confidence level class mark to point $\mathbf{x}$ in the input feature space [28]:

$$
f: \mathfrak{R}^{n} \rightarrow[0, g]^{m}
$$

The basis of the fuzzy rule-based classifier is the database of fuzzy production rules of the following form:

$R_{j}$ : IF $s_{1} \wedge x_{1}=A_{j 1}$ AND $s_{2} \wedge x_{2}=A_{j 2}$ AND ... AND $s_{n} \wedge x_{n}=A_{j n}$ THEN class $=c_{j}$

where $A_{j i}$ is the fuzzy term describing the $i$-th feature in the $j$ th rule $(j=\overline{1, R}, \quad i=\overline{1, n}) ; s_{i} \wedge x_{i}$ indicates the presence $\left(s_{i}=\right.$ $1)$ or absence $\left(s_{i}=0\right)$ of the feature in the classifier; $R$ is the number of rules. In this paper, we chose to make the number of rules equal to the number of classes in order to reduce the computational complexity; subsequently, $R=m$.

The fuzzy term indicates the function of attribution of a feature to a class. We used the fuzzy Gaussian terms that have two parameters: $b$ is the $x$-coordinate of the vertex, $c$ is the scatter. The membership function of the Gaussian terms is set as follows:
$A_{j i}=\exp \left(-\left(\frac{x_{i}-b_{j i}}{c_{j i}}\right)^{2}\right)$.

The term parameters for all features comprise the system antecedents' vector $\boldsymbol{\theta}=\left(b_{11}, c_{11}, b_{12}, c_{12}, b_{13}, c_{13}, b_{21}, c_{21}, \ldots\right.$, $\left.b_{n m}, c_{n m}\right)$. Figure 1 shows an example of partitioning a certain feature by four Gaussian terms.

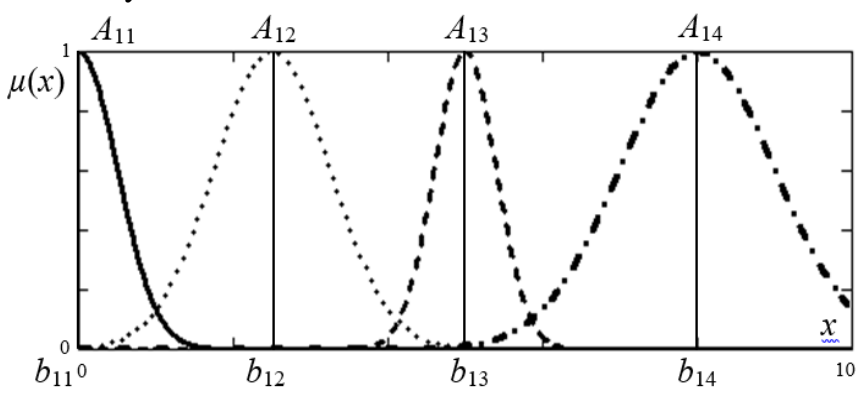

Fig. 1. Example of the fuzzy partition of feature $x$ by four Gaussian terms. The membership function $\mu(x)$ takes values from zero to one and shows the degree of membership of the point to the term

For the input vector $\left(x_{1}, x_{2}, \ldots, x_{n}\right)$, its membership in each rule is evaluated:

$\beta_{j}=\prod_{i=1}^{n} \mu_{A_{j i}}\left(x_{i}\right)$

where $\mu_{A_{j i}}\left(x_{i}\right)$ is the value of the function of fuzzy term $A_{k i}$ membership at point $x_{k}$. The output class is the class whose rule has the highest membership degree:

class $=\arg \max _{1 \leq i \leq m} \beta_{j}$.

\section{B. Performance of the fuzzy classifiers}

The most common classification quality criterion is the generalized accuracy that represents the percentage of correct classification. The accuracy measure can be set on the observation table $\left\{\left(\mathbf{x}_{p} ; c_{p}\right), p=\overline{1, z}\right\}$ as follows:

$E(\boldsymbol{\theta}, \mathbf{S})=\frac{\sum_{p=1}^{z}\left\{\begin{array}{c}1, \text { if } c_{p}=\arg \max f\left(x_{p} ; \boldsymbol{\theta} ; \mathbf{S}\right) \\ 0, \text { else }\end{array}\right.}{z}$,

where $f\left(\mathbf{x}_{p} ; \boldsymbol{\theta}, \mathbf{S}\right)$ is the output of the fuzzy rule-based classifier with the term parameter vector $\boldsymbol{\theta}$ and the binary vector of features $\mathbf{S}$ at point $\mathbf{x}_{p}$.

Another measure of the quality of the built system is the geometric mean applied in case of an imbalance in the number of class instances [29]:

$\operatorname{GM}(\boldsymbol{\theta}, \mathbf{S})=\left(\prod_{i=1}^{m} A c c_{i}(\boldsymbol{\theta}, \mathbf{S})\right)^{\frac{1}{m}}$,

where $A c c_{i}(\boldsymbol{\theta}, \mathbf{S})$ is the classification accuracy of the $i$-th class.

In case there is a large number of features and classes in addition to the class imbalance, a more sophisticated criterion of evaluation is required in order to evaluate the model quality. In this paper, it is proposed to use the statistical Akaike information criterion [30] adapted to evaluate the quality of the built classifier based on the geometrical mean value and the ratio of the number of features in the subset found by the algorithm and the original set: 


$$
A I C(\boldsymbol{\theta}, \mathbf{S})=\ln (1-G M(\boldsymbol{\theta}, \mathbf{S}))+\frac{c F}{n},
$$

where $F$ is the number of features in the selected subset, $n$ is the number of features in the full set, $c$ is the priority coefficient: if $c<1$, the part of the expression related to accuracy is more important, while if $c>1$, the ratio of the number of found features and the original number is more important. At the same time, if $c=1$, the two terms are equally important.

The problem of building a fuzzy rule-based classifier is reduced to searching for the extreme point of the target function (the maximum for $\mathrm{E}(\boldsymbol{\theta}, \mathbf{S})$ and $\operatorname{GM}(\boldsymbol{\theta}, \mathbf{S})$, the minimum for $\operatorname{AIC}(\boldsymbol{\theta}, \mathbf{S}))$ in space $\mathbf{S}$ and $\boldsymbol{\theta}=\left(\theta^{1}, \theta^{2}, \ldots, \theta^{D}\right)$.

The formation of a fuzzy rule-based classifier includes three stages: structure generation, feature selection, and tuning fuzzy rule parameters. At the stage of structure generation, the algorithm for generating rule base by extreme feature values creates fuzzy terms and the rule database, thus initializing the original $\boldsymbol{\theta}$ and $\mathbf{S}$ vectors. Further, the binary Gravitational Search Algorithm with constant vector $\boldsymbol{\theta}$ searches for such vector $\mathbf{S}$ that enhances the classification quality. Upon the binary algorithm completion with the selected feature vector $\mathbf{S}_{\text {best }}$, the parameters of the vector $\boldsymbol{\theta}$ are selected with the continuous Gravitational Search Algorithm. As the Gravitational Search Algorithm works as a wrapper, each change in any of the vectors results in reorganization of the entire classifier in order to evaluate the decision quality.

\section{GENERALIZED FuZZY RULE-BASED ClASSIFIER STRUCTURE}

The objective of the algorithm for generating the structure of a fuzzy classifier is to create a base of fuzzy rules and fuzzy terms for each feature. To create a compact rule base, it is proposed to use an algorithm based on class extremes, which allows using a minimum number of rules equal to the number of classes [31].

The algorithm consists in the following. The space of feature $x_{i}$ is partitioned in $m$ subsets $x_{i}=\left\{x_{i}^{1}, x_{i}^{2}, \cdots, x_{i}^{m}\right\}$, each of which corresponds to a class of the entire set of classes. Next, the greatest and smallest values of the feature are searched in the subsets for each class. Based on this values the covering Gaussian term is built. Thus, the number of terms for each feature is equal to the number of classes. The pseudo code of the algorithm for generating rule base by extreme feature values is provided in [32].

\section{FEATURE SELECTION AND TUNING FUZZY RULE PARAMETERS WITH THE GRAVITATIONAL SEARCH ALGORITHM}

Problems of feature selection and finding the best system parameters can be solved by optimization. In our work, we use the gravitational search algorithm in two versions: binary to form a subset of relevant features and continuous to search for the best parameters of terms.

The binary Gravitational Search Algorithm is used to search such a subset of features, which would not lead to a reduction in the classification accuracy with a decrease in the number of features. The algorithm operates binary vectors $\mathbf{S}=\left(s_{1}, s_{2}, \ldots\right.$, $\left.s_{n}\right)^{\mathrm{T}}$ in the wrapper mode. The dimension of each vector $\mathbf{S}$ is equal to the original number of features; $s_{i}=0$ means that the $i$-th feature is not included in the classification, $s_{i}=1$ means that the $i$-th feature is used by the classifier. The original population of binary vectors $\mathbf{S}=\left\{\mathbf{S}_{1}, \mathbf{S}_{2}, \ldots, \mathbf{S}_{P}\right\}$ is set randomly. The vector quality is estimated by the target function, for which evaluation it is required to construct the classifier.

The algorithm calculates a number of physical characteristics at each iteration: masses, accelerations and velocities of particles. The vector's elements are updated by transforming the numerical value of the speed into its binary equivalent with the transform function, which determines the probability of changes in the value of the vector's element to the opposite one [33]. The output of the algorithm is vector $\mathbf{S}_{\text {best }}$ with the best value of the target function.

The continuous algorithm is required in order to optimize the vector of the antecedents' parameters of the fuzzy rulebased system $\theta$. The population of vectors $\Theta=\left\{\theta_{1}, \theta_{2}, \ldots, \theta_{P}\right\}$ is created based on the input vector $\theta_{1}$, obtained by the classifier structure generation algorithm. Then the same calculations occur as in the binary version of the algorithm, but elements of each vector $\boldsymbol{\theta}$ are updated by adding to their current speed value. A detailed description of the algorithms and their pseudo-codes are presented by us in [32].

\section{EXPERIMENT}

The data for constructing classifiers implementing the verification procedure was prepared as follows. In total, eight users were involved. Legitimate user inputted handwritten signature on the graphic tablet. Others tried to falsify his signature. The number of examples of each user's signatures varied between 119 and 280; in total, the database contained 1559 signatures. Before extracting the features, each obtained signature was preprocessed. The preprocessing consisted in correcting technical errors, bringing the signature orientation to a standard form, bringing it to a single scale and finding the exact moment of the beginning and completion of a signature.

During the experiment, the data for the handwritten signature analysis system were obtained with a WACOM graphic tablet. It was used to form the following dynamic sequences of the discrete time: 1) pen location relatively to the $x, y, z$ axes; 2) pen pressure $P$; 3) azimuth $\alpha$;) pen height angle $\theta$ relatively to the tablet. The observation dataset was created from the first eight harmonics of the specified sequences' decomposition into a Fourier series [34]. Thus, each entry of the observation table was a description of the handwritten signature with 144 features and the class mark being the signing user's number. The parameters and the enumerated features formed based on them are provided in Table 1 , here $H_{i}$ is number of $i$-th harmonic.

The experiment consisted of two stages. At the first stage, it was required to verify the effectiveness of constructing the fuzzy rule-based classifier for the handwritten signature's authenticity verification. In this case, if the database instance belonged to the legitimate user, the instance was assigned 
class " 1 "; if the signature belonged to one of the seven intruders, it was assigned class " 0 ." The dataset created based on the 2-class database was designated as Signature2.

The experiment was performed in accordance with the cross-validation scheme as follows: ten database duplicates were created, and then each duplicate was divided into a training sample and a test sample in a 9:1 ratio. At the same time, data from a test sample of one duplicate should not overlap with data from test samples of other duplicates.

Further, each data duplicate underwent the following procedures:

1) The fuzzy rule-based classifier with two production rules and Gaussian terms was constructed on the training sample by the algorithm for generating rule base by extreme feature values.

2) The binary Gravitational Search Algorithm was run five times for the constructed classifier; since the algorithm is stochastic, five different sets of features could be received.

3) Among the obtained feature sets, we chose the one with the best value of the target function. In case of equal values,

the set with a smaller number of features was chosen.

TABLE I.

LIST OF DYNAMIC FEATURES OF THE HANDWRITTEN SIGNATURE.

\begin{tabular}{lllllllll}
\hline \hline Parameter & $H_{1}$ & $H_{2}$ & $H_{3}$ & $H_{4}$ & $H_{5}$ & $H_{6}$ & $H_{7}$ & $H_{8}$ \\
\hline Parameter $x$ & 1 & 2 & 3 & 4 & 5 & 6 & 7 & 8 \\
Parameter $y$ & 9 & 10 & 11 & 12 & 13 & 14 & 15 & 16 \\
Parameter $z$ & 17 & 18 & 19 & 20 & 21 & 22 & 23 & 24 \\
Parameter $P$ & 25 & 26 & 27 & 28 & 29 & 30 & 31 & 32 \\
Parameter $\alpha$ & 33 & 34 & 35 & 36 & 37 & 38 & 39 & 40 \\
Parameter $\theta$ & 41 & 42 & 43 & 44 & 45 & 46 & 47 & 48 \\
Speed $x$ & 49 & 50 & 51 & 52 & 53 & 54 & 55 & 56 \\
Speed $y$ & 57 & 58 & 59 & 60 & 61 & 62 & 63 & 64 \\
Speed $z$ & 65 & 66 & 67 & 68 & 69 & 70 & 71 & 72 \\
Speed $P$ & 73 & 74 & 75 & 76 & 77 & 78 & 79 & 80 \\
Speed $\alpha$ & 81 & 82 & 83 & 84 & 85 & 86 & 87 & 88 \\
Speed $\theta$ & 89 & 90 & 91 & 92 & 93 & 94 & 95 & 96 \\
Acceleration $x$ & 97 & 98 & 99 & 100 & 101 & 102 & 103 & 104 \\
Acceleration $y$ & 105 & 106 & 107 & 108 & 109 & 110 & 111 & 112 \\
Acceleration $z$ & 113 & 114 & 115 & 116 & 117 & 118 & 119 & 120 \\
Acceleration $P$ & 121 & 122 & 123 & 124 & 125 & 126 & 127 & 128 \\
Acceleration $\alpha$ & 129 & 130 & 131 & 132 & 133 & 134 & 135 & 136 \\
Acceleration $\theta$ & 137 & 138 & 139 & 140 & 141 & 142 & 143 & 144 \\
\hline \hline
\end{tabular}

TABLE II.

THE RESULTS OF THE FUZZY CLASSIFIERS BASED ON THE SIGNATURE2 DATASET.

\begin{tabular}{|c|c|c|c|c|c|c|c|c|}
\hline$N$ & $F$ & $E_{\mathrm{tr}}$ & $G M_{t r}$ & $E_{t s t}$ & $G M_{t s t}$ & $E_{1 t s t}$ & $E_{2 t s t}$ & Features \\
\hline 1 & 6 & 99.84 & 99.88 & 99.62 & 99.48 & 0.71 & 0.31 & $4,5,17,33,74,140$ \\
\hline 2 & 5 & 99.75 & 99.83 & 99.42 & 99.22 & 1.07 & 0.47 & $2,33,36,61,142$ \\
\hline 3 & 4 & 99.94 & 99.95 & 99.74 & 99.56 & 0.71 & 0.16 & $18,33,64,143$ \\
\hline 4 & 6 & 99.86 & 99.92 & 99.74 & 99.70 & 0.36 & 0.23 & $14,23,33,47,126,144$ \\
\hline 5 & 4 & 99.91 & 99.95 & 99.81 & 99.74 & 0.36 & 0.16 & $5,33,48,97$ \\
\hline 6 & 3 & 100 & 100 & 99.87 & 99.78 & 0.36 & 0.08 & $22,33,144$ \\
\hline 7 & 8 & 99.94 & 99.97 & 99.81 & 99.74 & 0.36 & 0.16 & $11,22,33,47,48,56,105,144$ \\
\hline 8 & 3 & 99.87 & 99.92 & 99.74 & 99.84 & 0 & 0.31 & $18,33,143$ \\
\hline 9 & 5 & 99.84 & 99.89 & 99.62 & 99.34 & 1.07 & 0.23 & $4,13,33,67,121$ \\
\hline 10 & 3 & 99.91 & 99.93 & 99.81 & 99.74 & 0.36 & 0.16 & $22,33,143$ \\
\hline Avg. & 4.7 & 99.89 & 99.92 & 99.72 & 99.62 & 0.54 & 0.23 & \\
\hline
\end{tabular}

4) The classifier based on the selected set of features was constructed for each of the ten training samples.

5) The built classifiers were trained by the continuous Gravitational Search Algorithm.

6) Upon training, the classifiers were verified using the test sample, the percentage of type I and II errors were calculated.

7) The values of the target function, accuracy, and errors were averaged by the number of samples.

Thus, the binary Gravitational Search Algorithm selected ten feature sets. In this case, the geometrical mean was the target function of the binary and continuous Gravitational Search Algorithms.

The results of the constructed fuzzy rule-based classifiers are provided in Table 2. Here, $N$ is the sample number, for which the given feature set was obtained; $F$ is the number of features in the set; $E_{t r}$ is the percentage of correct classification, averaged by ten training samples; $G M_{t r}$ is the geometrical mean in percentage, averaged by ten training samples; $E_{t s t}$ is the percentage of correct classification, averaged by ten test samples; $G M_{t s t}$ is the geometrical mean in percentage, averaged by ten test samples; $E_{1 t s t}$ and $E_{2 t s t}$ is the type I and II error percentage, respectively.

At the first stage of the experiment, the parameters were as follows. Binary Gravitational Search Algorithm: 250 iterations, 10 particles, the initial value of the gravitational constant $G_{0}=10$, coefficient $\alpha=10$, small constant $\varepsilon=0.01$. Continuous Gravitational Search Algorithm: 500 iterations, 10 particles, the initial value of the gravitational constant $G_{0}=10$, coefficient $\alpha=10$, small constant $\varepsilon=0.01$. These values have been empirically chosen.

At the second stage of the experiment, the same data was used, but the intruder's signature instance was assigned the class corresponding to the intruder's number. Thus, the number of classes and fuzzy rules was equal to eight; 
therefore, such dataset version was called Signature8. This stage was aimed at verifying the ability of the classifier to not only detect if the signature was veritable, but also identify the particular intruder.

The target function for the binary Gravitational Search Algorithm was the adapted Akaike information criterion, and for the continuous Gravitational Search Algorithm used the geometrical mean. The second stage of the experiment was performed in the same order, except for stage 4, as the best set of features was selected based on the value of the Akaike information criterion.

TABLE III.

THE RESULTS OF THE FUZZY RULE-BASED CLASSIFIERS BASED ON THE SIGNATURE8 DATASET.

\begin{tabular}{|c|c|c|c|c|c|c|c|c|c|c|c|c|}
\hline$N$ & $E_{\mathrm{tr}}$ & $E_{\mathrm{tst}}$ & $F$ & $A I C$ & $A_{1}$ & $A_{2}$ & $A_{3}$ & $A_{4}$ & $A_{5}$ & $A_{6}$ & $A_{7}$ & $A_{8}$ \\
\hline 1 & 96.72 & 95.54 & 31 & -3.0 & 99.2 & 93.8 & 95.4 & 97.1 & 97.4 & 99.4 & 91.6 & 97.8 \\
\hline 2 & 93.67 & 91.67 & 12 & -2.4 & 99.6 & 95.1 & 94.4 & 97.1 & 97.4 & 97.8 & 71.0 & 87.0 \\
\hline 3 & 96.73 & 94.23 & 26 & -3.0 & 100 & 96.5 & 98.0 & 87.1 & 98.3 & 99.4 & 94.4 & 96.2 \\
\hline 4 & 94.95 & 96.79 & 20 & -2.6 & 97.6 & 94.7 & 94.9 & 86.4 & 98.3 & 100 & 83.2 & 97.8 \\
\hline 5 & 96.23 & 92.31 & 19 & -3.0 & 99.2 & 95.6 & 95.4 & 90.0 & 99.1 & 97.2 & 93.5 & 97.3 \\
\hline 6 & 93.17 & 92.95 & 17 & -2.3 & 99.2 & 95.2 & 91.3 & 94.3 & 98.3 & 98.9 & 72.9 & 86.5 \\
\hline 7 & 96.51 & 94.23 & 20 & -2.9 & 98.4 & 95.6 & 98.0 & 98.6 & 97.4 & 99.4 & 81.5 & 97.3 \\
\hline 8 & 94.59 & 96.79 & 26 & -2.6 & 97.2 & 94.7 & 89.3 & 95.7 & 90.6 & 98.3 & 93.5 & 95.1 \\
\hline 9 & 95.59 & 93.59 & 13 & -2.9 & 97.2 & 94.7 & 97.4 & 94.3 & 98.3 & 93.4 & 86.9 & 98.9 \\
\hline 10 & 96.16 & 94.87 & 22 & -2.9 & 99.2 & 95.2 & 96.4 & 92.9 & 99.1 & 99.4 & 86.9 & 95.7 \\
\hline Avg. & 95.43 & 94.30 & 20.6 & -2.8 & 98.7 & 95.1 & 95.1 & 93.4 & 97.4 & 98.3 & 85.5 & 95.0 \\
\hline
\end{tabular}

The results of the created fuzzy classifiers, which were trained by the continuous Gravitational Search Algorithm, are provided in Table 3. Here, $N$ is the sample number, for which the given feature set was obtained; $F$ is the number of features in the set; $E_{t r}$ is the percentage of correct classification, averaged by ten training samples; $E_{t s t}$ is the percentage of correct classification, averaged by ten test samples; $A I C$ is the averaged Akaike factor, averaged by ten training samples; $A_{k}$ is the percentage of correct classification of the $k$-th class, $k=\overline{1,8}$.

At the second stage of the experiment, the algorithms' parameters were as follows: Binary Gravitational Search Algorithm: 500 iterations, 10 particles, the initial value of the gravitational constant $G_{0}=10$, coefficient $\alpha=10$, small constant $\varepsilon=0.01$, factor for finding the Akaike criterion $c=$ 1.5. Continuous Gravitational Search Algorithm: 250 iterations, 10 particles, the initial value of the gravitational constant $G_{0}=10$, coefficient $\alpha=10$, small constant $\varepsilon=0.01$. These values have been empirically chosen.

\section{RESULTS AND ANALYSIS}

Using the Gravitational Search Algorithm to construct the fuzzy rule-based classifier allows significantly reducing the number of features and improving accuracy. Table 4 shows a comparison of the results demonstrated by 1) the fuzzy rulebased classifier built for a full dataset by the algorithm for generating rule base by extreme feature values without training, 2) the fuzzy rule-based classifier built based on the algorithm for generating rule base by extreme feature values, with features selected by the binary Gravitational Search Algorithm and the terms tuned by the continuous Gravitational Search Algorithm.
The results showed that in the Signature 2 dataset, the number of features reduced by almost $97 \%$ and the averaged accuracy improved by $88 \%$, as compared to the full set of features. For the Signature8 dataset, there was almost zero accuracy improvement, but the number of features reduced by approximately $85.7 \%$. Besides, for the Signature2 dataset, we managed to create the classifier with almost $100 \%$ training accuracy and $99.87 \%$ testing accuracy for just three features (No.6 in Table 2).

TABLE IV.

COMPARISON OF THE NUMBER OF FEATURES AND PERCENTAGE OF CORRECT CLASSIFICATION FOR THE NON-TRAINED FUZZY RULE-BASED CLASSIFIER AND THE FUZZY RULE-BASED CLASSIFIER TRAINED BY THE GRAVITATIONAL SEARCH ALGORITHM.

\begin{tabular}{lllll}
\hline \hline Algorithm & Dataset & $F$ & $E_{t r}$ & $E_{t s t}$ \\
\hline Fuzzy classifier & Signature2 & 144 & 52.91 & 52.92 \\
Fuzzy classifier with GSA & Signature2 & 4.7 & 99.89 & 99.72 \\
Fuzzy classifier & Signature8 & 144 & 93.35 & 92.89 \\
Fuzzy classifier with GSA & Signature8 & 20.6 & 95.43 & 94.30 \\
\hline \hline
\end{tabular}

At the first stage of the experiment, Feature No.33 was included in all feature sets. This proves the consistency of the results of the binary Gravitational Search Algorithm and allows stating that the azimuth is the distinctive characteristic of the legitimate user. At the second stage, there were no features common for all sets. This is due to the fact that the classifier should have not only detected whether the signature belonged to the legitimate user, but also to identify the particular intruder.

In order to prove the effectiveness of the fuzzy rule-based classifier, a comparison was made using the most common classification algorithms presented in Scikit-learn (machine learning library for the Python) to verify the effectiveness of the fuzzy classifier. The following algorithms were used: gaussian naive Bayes classifier (GNBC), logistic regression 
classifier (LRC), decision tree classifier (DTC), multi-layer perceptron classifier (MLPC), linear support vector classifier (LSVC), k-nearest neighbors classifier with $\mathrm{k}=3$ (3NN), AdaBoost classifier $(\mathrm{ABC})$, random forest classifier (RFC), gradient boosting for classification (GB), and linear SVM with stochastic gradient descent training (SGD). All algorithm parameters are taken by default [35].
The average values of classification accuracy and geometric mean over ten test samples of the Signature2 data set are presented in Table 5.

The fuzzy rule-based classifier (FRBC) is second only to multi-layer perceptron classifier in accuracy and it is superior to other algorithms by the value of the geometric mean.

TABLE V.

THE RESULTS OF CLASSIFIERS BASED ON THE SIGNATURE2 DATASET.

\begin{tabular}{|c|c|c|c|c|c|c|c|c|c|c|c|}
\hline & GNBC & LRC & DTC & MLPC & LSVC & $3 \mathrm{NN}$ & $\mathrm{ABC}$ & RFC & GB & SGD & FRBC \\
\hline$E_{\mathrm{tst}}$ & 56,38 & 99,36 & 99,10 & 99,74 & 99,04 & 99,17 & 98,91 & 99,23 & 99,36 & 99,23 & 99,72 \\
\hline$G M_{t s t}$ & 61,23 & 99,17 & 98,60 & 99,27 & 98,98 & 97,70 & 99,18 & 98,11 & 98,74 & 98,10 & 99,62 \\
\hline
\end{tabular}

The computational complexity of the Gravitational Search Algorithm is of $\mathrm{O}(n \times d)$ where $n$ is the number of agents and $d$ is the search space dimension [36]. The Gravitational Search Algorithm in our work has not been modified, so it has complexity $\mathrm{O}(P \times d)$, where $P$ is the number of particles and $d$ is the size of the dataset.

\section{CONCLUSION}

The study has shown that the fuzzy rule-based classifier can be used as an element of the authentication system as a component of the handwritten signature verification based on dynamic features. The binary Gravitational Search Algorithm reduces the number of features, helps find the most relevant characteristics and eventually simplify the system. As a result, the number of required calculations in the end software product reduces. The continuous Gravitational Search Algorithm allows improving the classification accuracy.

\section{REFERENCES}

[1] J. Wayman, A. Jain, D. Maltoni, and D. Maio, "An Introduction to Biometric Authentication Systems," in Biometric Systems, J. Wayman, A. Jain, D. Maltoni, and D. Maio, eds., Springer, London, 2005, pp.120, 10.1007/1-84628-064-8 1 .

[2] K. Cao and A. K. Jain, "Hacking mobile phones using 2D printed fingerprints," Michigan State University, MSU-CSE-16-2, Feb. 2016.

[3] R. Tronci et al. "Fusion of multiple clues for photo-attack detection in face recognition systems," in Proc. Biometrics, International Joint Conference, Washington, USA, 2011, pp. 1-6, DOI: 10.1109/IJCB.2011.6117522.

[4] S. S. Mudholkar, P. M. Shende, and M. V.Sarode, "Biometrics Authentication Technique for Intrusion Detection Systems Using Fingerprint Recognition," I. J. of Computer Science, Engineering and Information Technology, vol. 2, no. 4, pp. 57-65, Feb. 2012, DOI: 10.5121/ijcseit.2012.2106.

[5] X. Hu, W. Pedrycz, and X. Wang, "Fuzzy classifiers with information granules in feature space and logic-based computing," Pattern Recognit., vol. 80, pp. 156-167, August 2018, DOI: 10.1016/j.patcog.2018.03.011.

[6] P. K. Shukla and S. P. Tripathi, "Interpretability and accuracy issues in evolutionary multi-objective fuzzy classifiers," I. J. of Soft Computing and Networking, vol. 1, no. 1, pp. 55-69, June 2016, DOI: 10.1504/IJSCN.2016.077043.

[7] A. E. Yankovskaya, I. V. Gorbunov, and I. A. Hodashinsky, "Tradeoff search methods between interpretability and accuracy of the identification fuzzy systems based on rules," Pattern Recognit. Image Anal., vol. 27, no. 2, pp. 243-265, June 2017, DOI: 10.1134/S1054661817020134.

[8] S.-B. Roh, W. Pedrycz, and T.-C. Ahn, "A design of granular fuzzy classifier," Expert Syst. Appl., vol. 41, no. 15, pp. 6786-6795, November 2014, DOI: 10.1016/j.eswa.2014.04.040.
[9] Y. Torun and G. Tohumoglu, "Designing simulated annealing and subtractive clustering based fuzzy classifier," Appl. Soft Comput., vol. 11, pp. 2193-2201, March 2015, DOI: 10.1016/j.asoc.2010.07.020.

[10] A. Lavygina and I. Hodashinsky, "Hybrid algorithm for fuzzy model parameter estimation based on genetic algorithm and derivative based methods," in Proc. ECTA 2011 FCTA 2011, pp. 513-515.

[11] S. Garcia-Galan, R. P. Prado, and J.E. M. Expósito, "Rules discovery in fuzzy classifier systems with PSO for scheduling in grid computational infrastructures," Appl. Soft Comput., vol. 29, pp. 424-435, April 2015, DOI: 10.1016/j.asoc.2014.11.064.

[12] E. Zhou and A. Khotanzad, "Fuzzy classifier design using genetic algorithms," Pattern Recognit., vol. 40, pp. 3401-3414, December 2007, DOI: 10.1016/j.patcog.2007.03.028.

[13] I. A. Hodashinsky, D.Y. Minina, and K.S. Sarin, "Identification of the parameters of fuzzy approximators and classifiers based on the cuckoo search algorithm," Optoelectronics, Instrumentation and Data Processing, vol. 51, no. 3, May 2015, pp. 234-240, DOI: $10.3103 /$ S8756699015030048.

[14] A. M. Acilar and A. Arslan, "A novel approach for designing adaptive fuzzy classifiers based on the combination of an artificial immune network and a memetic algorithm," Inf. Sci., vol. 264, pp. 158-181, April 2014, DOI: 10.1016/j.ins.2013.12.023.

[15] G. Gupta and A. McCabe, "A Review of Dynamic Handwritten Signature Verification," James Cook University, Computer Science Dept., Technical report at James Cook University, Sept. 1997.

[16] M. Zalasinski, K. Cpalka, and L. Rutkowski, "Fuzzy-Genetic Approach to Identity Verification Using a Handwritten Signature," Studies in Computational Intelligence, vol. 738, pp. 375-394, Jan. 2018, DOI: 10.1007/978-3-319-67946-4_17.

[17] A. Sharma and S. Sundaram, "An enhanced contextual DTW based system for online signature verification using Vector Quantization," Pattern Recognit. Lett., vol. 84, pp. 22-28, July 2016, DOI: 10.1016/j.patrec.2016.07.015.

[18] A. Kholmatov and B. Yanikoglu, "Identity authentication using improved online signature verification method," Pattern Recognit. Lett., vol. 26, pp. 2400-2408, Nov. 2005, DOI: 10.1016/j.patrec.2005.04.017.

[19] X. Xia, Z. Chen, F. Luan, and X. Song, "Signature alignment based on GMM for on-line signature verification," Pattern Recognit., vol. 65, pp. 188-196, May 2017, DOI: 10.1016/j.patcog.2016.12.019.

[20] M.A.U. Khan, M.K. Khan, and M.A. Khan, "Velocity-image model for online signature verification," IEEE Trans. Image Processing, vol. 15, no. 11, pp. 3540-3549, Dec 2006, DOI: 10.1109/TIP.2006.877517.

[21] K. Cpalka, M. Zalasinski, and L. Rutkowski, "A new algorithm for identity verification based on the analysis of a handwritten dynamic signature," Appl. Soft Comput., vol. 43, pp. 47-56, June 2016, DOI: 10.1016/j.asoc.2016.02.017

[22] M. I. Razzak and B. Alhaqbani, "Multilevel fusion for fast online signature recognition using multi-section VQ and time modelling," Neural Computing and Applications, vol. 26, no. 5, pp. 1117-1127, Dec. 2014, DOI: 10.1007/s00521-014-1779-6.

[23] R. Doroz, P. Porwik, and T. Orczyk, "Dynamic signature verification method based on association of features with similarity measures," Neurocomputing, vol. 171, pp. 921-931, July 2015, DOI: 10.1016/j.neucom.2015.07.026.

[24] Nanni L, Maiorana E, Lumini A, and P.Campisi, "Combining local, regional and global matchers for a template protected on-line signature 
verification system,” Expert Syst. Appl., vol. 37, no. 5, pp. 3676-3684, May 2010, DOI: 10.1016/j.eswa.2009.10.023.

[25] R. Kohavi and G. H. John, "Wrappers for feature subset selection," Artif. Intell., vol. 97, no. 1, pp. 273-324, Dec 1997, DOI: 10.1016/S0004-3702(97)00043-X

[26] V. Bolon-Canedo, N. Sanchez-Marono, and A. Alonso-Betanzos, Feature Selection for High-Dimensional data. Springer, Heidelberg, 2015.

[27] E. Rashedi, H. Nezamabadi-pour, and S. Saryazdi, "GSA: A Gravitational Search Algorithm," Inf. Sci., vol. 179, pp. 2232-2248, June 2009, DOI: 10.1016/j.ins.2009.03.004.

[28] I. A. Hodashinsky and M. A.Mekh, "Fuzzy classifier design using harmonic search methods," Program. Comput. Soft., vol. 43, no. 1, pp. 37-46, Jan. 2017, DOI: 10.1134/S0361768817010030.

[29] J. A. Sanz, D. Bernardo, F. Herrera, H. Bustince, and H. Hagras, "A Compact Evolutionary Interval-Valued Fuzzy Rule-Based Classification System for the Modeling and Prediction of Real-World Financial Applications with Imbalanced Data," IEEE Trans. on Fuzzy Syst., vol. 23, no. 4. pp. 973 - 990, Aug. 2015, DOI: 10.1109/TFUZZ.2014.2336263

[30] J. Yen and L. Wang, "Application of Statistical Information Criteria for Optimal Fuzzy Model Construction," IEEE Trans. Fuzzy Syst., vol. 6, no. 3. pp. 362-372, Sep. 1998, DOI: $10.1109 / 91.705503$.

[31] M. A. Mekh and I. A.Hodashinsky, "Comparative analysis of differential evolution methods to optimize parameters of fuzzy classifiers," J. Comput. Syst. Sci. Int., vol. 56, no. 4, pp. 616-626, 2017, DOI: $10.1134 / \mathrm{S} 1064230717040116$.

[32] M. Bardamova, A. Konev, I. Hodashinsky, and A. Shelupanov, "A Fuzzy Classifier with Feature Selection Based on the Gravitational Search Algorithm," Symmetry, vol. 10, p. 609, Nov. 2018, DOI: 10.3390/sym10110609.

[33] E. Rashedi, H. Nezamabadi-pour, and S. Saryazdi, "BGSA: binary gravitational search algorithm," Nat. Comput., vol. 9, no. 3, pp. 727745, Sep. 2010, DOI: 10.1007/s11047-009-9175-3.

[34] E. Kostyuchenko et al. "Integration of Bayesian classifier and perceptron for problem identification on dynamics signature using a genetic algorithm for the identification threshold selection," Lecture Notes in Computer Science, vol. 9719, pp. 620-627, July 2016, DOI 10.1007/978-3-319-40663-3_71.

[35] Supervised learning. User Guide. Scikit-learn. Machine Learning in Python (2019, July 29). [Online]. Available: https://scikitlearn.org/stable/supervised_learning.html\#supervised-learning

[36] D. Pelusi, R. Mascella, L. Tallini, J. Nayak, B. Naik, and A. Abraham, "Neural network and fuzzy system for the tuning of Gravitational Search Algorithm parameters," Expert Syst. Appl. vol. 102, pp. 234 244, Jul. 2018,doi:10.1016/j.eswa.2018.02.026.

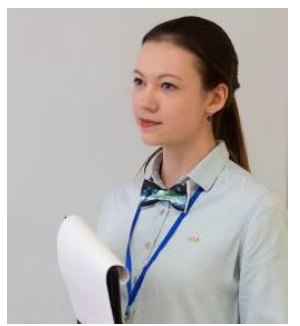

Marina Bardamova. She was born in 1995 , graduated from the Faculty of Security, Tomsk State University of Control Systems and Radioelectronics (TUSUR) in 2017. She is a junior researcher in TUSUR. Her main research interests include the computational intelligence, data mining, fuzzy modeling and machine learning. She is the author and co-author of over 10 journal and conference papers.

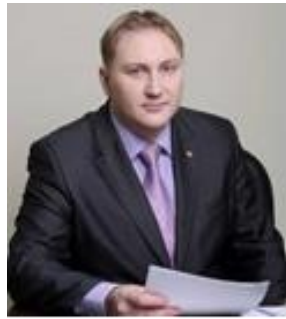

Anton Konev. He was born in 1982, graduated from the Faculty of Computing systems, Tomsk State University of Control Systems and Radioelectronics (TUSUR) in 2004

He is an Associate Professor at TUSUR. Received the $\mathrm{PhD}$ degree from the TUSUR in 2007. His main research interests include the information security, system modeling and speech recognition. $\mathrm{He}$ is the author and co-author of over 80 journal and conference papers.

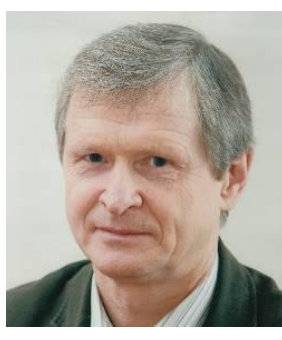

Ilya Hodashinsky. He was born in 1953 graduated from the Faculty of Control Systems, Novosibirsk Electrotechnical Institute (NETI) in 1975. Received the $\mathrm{PhD}$ degree in 1984 , the Dr.Sc. degree in 2004 from the Tomsk State University of Control Systems and Radioelectronics (TUSUR), Russia. Received the Professor title at the 2011

$\mathrm{He}$ is a professor TUSUR. His main research interests include the computational intelligence, fuzzy modeling, pattern recognition, knowledge discovery, and data mining. He is the author and co-author of over 170 journal and conference papers as well as technical articles. Prof. Hodashinsky is a member of IEEE, IEEE Computational Intelligence Society.

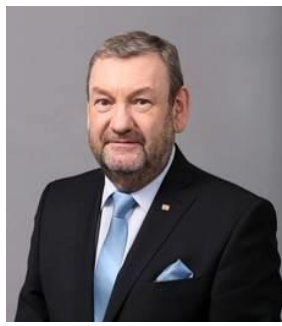

Alexander Shelupanov. He was born in 1954, graduated from Tomsk State University in the following specialties: "Applied Mathematics" and "Mechanics" in 1976. He has been working in Tomsk State University of Control Systems and Radioelectronics (TUSUR) since 1993.

In 2014 he was elected to the position of rector of TUSUR. In 1991 he became a candidate of physical and mathematical sciences. In 1996 he defended his thesis for the degree of Doctor of Technical Sciences. In 2003 he was awarded the title of professor, was elected a full member of the International Academy of Higher Education and the International Academy of Informatization. Scientific activity is related to the modeling of complex technical systems, fast processes, the creation of theoretical foundations, systems and methods of information protection, information security. He is the author of more than 400 publications, including 8 scientific monographs, 32 textbooks, three of which are classified by the Ministry of Education and Science of the Russian Federation. In the scientific school of Prof. A. Shelupanov there are more than 25 candidates and doctors of science prepared by him. 